\title{
FAMILIES OF LORENTZIAN MATRICES
}

C. C. MACDUfFEE

1. The differential equation. Let $P(s)$ be an $n$-rowed square matrix whose elements are continuous real functions of the real variable $s$ in an interval $s_{0} \leqq s \leqq s_{1}$. The differential equation

$$
\Lambda^{\prime}(s)=P(s) \cdot \Lambda(s), \quad \Lambda(s)=\left(\lambda_{i j}\right),
$$

is equivalent to the $n$ systems of equations

$$
\begin{aligned}
& \lambda_{1 i}^{\prime}=p_{11} \lambda_{1 i}+p_{12} \lambda_{2 i}+\cdots+p_{1 n} \lambda_{n i}, \\
& \lambda_{2 i}^{\prime}=p_{21} \lambda_{1 i}+p_{22} \lambda_{2 i}+\cdots+p_{2 n} \lambda_{n i}, \\
& \cdot \cdot \cdot \cdot \cdot \cdot \cdot \cdot \cdot \cdot \cdot \cdot \cdot \cdot \cdot \\
& \lambda_{n i}^{\prime}=p_{n 1} \lambda_{1 i}+p_{n 2} \lambda_{2 i}+\cdots+p_{n n} \lambda_{n i}, \quad i=1,2, \cdots, n .
\end{aligned}
$$

The coefficients are independent of $i$ so that each column of $\Lambda(s)$ is a solution of this system.

These equations are known $[1 ; 2 ; 3]^{1}$ to have a solution for every choice of initial conditions, and to have exactly $n$ linearly independent solutions, every solution then being a linear combination of any set of $n$ linearly independent ones. This is equivalent to the statement in matric notation that there is a solution $\Lambda(s)$ such that $\left|\Lambda\left(s_{0}\right)\right| \neq 0$, and that every solution is then given by $\Lambda(s) \cdot A$ where $A$ is an arbitrary constant real matrix. If the elements of $P(s)$ are $n-1$ times differentiable, it can be shown that each element of $\Lambda(s)$ satisfies a linear differential equation whose coefficients are polynomials in the elements of $P(s)$ and their derivatives.

If (1) has a solution $\Lambda(s)$ all of whose elements are analytic functions of $s$ in a neighborhood of $s=0$, it may be expressed in power series. From (1) we have

$$
\begin{aligned}
\Lambda^{\prime}(0) & =P(0) \cdot \Lambda(0), \\
\Lambda^{\prime \prime}(s) & =P^{\prime}(s) \cdot \Lambda(s)+P(s) \cdot \Lambda^{\prime}(s)=\left[P^{\prime}(s)+P^{2}(s)\right] \Lambda(s) .
\end{aligned}
$$

Similarly

$$
\Lambda^{(i)}(s)=M_{i}(s) \cdot \Lambda(s)
$$

where $M_{i}(s)$ is a polynomial in $P(s)$ and its derivatives. Then near

Presented to the International Congress of Mathematicians, September 2, 1950 under the title Curves in Minkowski space; received by the editors November 1, 1950.

1 Numbers in brackets refer to the references at the end of the paper. 
$s=0[4 ; 5]$

$$
\Lambda(s)=\left[I+M_{1}(0) s+\frac{1}{2} M_{2}(0) s^{2}+\frac{1}{3 !} M_{3}(0) s^{3}+\cdots\right] \Lambda(0) .
$$

In the case where $P$ is a constant matrix, this becomes particularly simple, for $\Lambda^{(i)}(s)=P^{i} \Lambda(s)$ so that

$$
\begin{aligned}
\Lambda(s) & =\left[I+P \cdot s+\frac{1}{2} P^{2} \cdot s^{2}+\frac{1}{3 !} P^{3} \cdot s^{3}+\cdots\right] \Lambda(0) \\
& =e^{P \cdot s} \Lambda(0),
\end{aligned}
$$

which is a known result. In fact, Hurewicz [3, p. 43] has shown that $\Lambda(s)=e^{\mathcal{S} P(s) \cdot d s} \Lambda(0)$ whenever $P(s)$ is commutative with its integral.

All the results of this section may be paralleled for the equation

$$
\Lambda^{\prime}(s)=\Lambda(s) \cdot Q(s)
$$

by taking the transposed matrices.

2. Lorentzian matrices. We shall denote by $J$ an $n$ by $n$ matrix whose elements are real numbers and which is both symmetric and orthogonal. Since $J$ is symmetric, there exists a real orthogonal matrix $O$ such that

$$
O^{T} J O=\left[\begin{array}{cccc}
\lambda_{1} & 0 & \cdots & 0 \\
0 & \lambda_{2} & \cdots & 0 \\
\cdot & \cdot & \cdot & \cdot \\
0 & 0 & \cdots & \lambda_{n}
\end{array}\right]=D
$$

where $\lambda_{1}, \lambda_{2}, \cdots, \lambda_{n}$ are the characteristic roots of $J$, and are all real. Since $J$ is orthogonal, these roots are of absolute value unity so that $\lambda_{i}= \pm 1$. Clearly

$$
J=J^{T}=J^{-1}, \quad|J|= \pm 1 .
$$

The set of all matrices $A$ such that

$$
A^{T} J A=J
$$

constitute a group which we shall call a Lorentzian group and denote by $R_{J}$. It is merely a matter of an isomorphism

$$
A \leftrightarrow O^{r} A O
$$

to suppose that $J$ is actually the diagonal matrix $D$. If $D=I$, then $\mathfrak{R}_{\boldsymbol{I}}$ is the orthogonal group of $n$ by $n$ matrices so that the theory of orthogonal matrices is contained in this treatment. 
Theorem 1. If $A \in \mathbb{R}_{J}$, then also $A^{T} \in \mathfrak{R}_{J}$.

For if $A^{T} J A=J$, then $A^{T} J A J=J^{2}=I$ so that

$$
A J=\left(A^{T} J\right)^{-1}=J^{-1} A^{-T}=J A^{-T} .
$$

That is, $A J A^{T}=J$.

THEOREM 2. Let $\Lambda(s)$ be a matrix whose elements are differentiable real functions of $s$ which is in $\ell_{J}$ for every $s$ in the interval $s_{0} \leqq s \leqq s_{1}$. Then there exist unique matrices $P(s)$ and $Q(s)$ such that $J \cdot P(s)$ and $Q(s) \cdot J$ are skew, and

$$
\Lambda^{\prime}(s)=P(s) \cdot \Lambda(s)=\Lambda(s) \cdot Q(s) .
$$

Upon differentiating $\Lambda^{T}(s) \cdot J \cdot \Lambda(s)=J$, we have

$$
\Lambda^{\prime T}(s) \cdot J \cdot \Lambda(s)+\Lambda^{T}(s) \cdot J \cdot \Lambda^{\prime}(s)=0 .
$$

That is,

$$
\begin{aligned}
\Lambda^{T}(s) \cdot P^{T}(s) \cdot J \cdot \Lambda(s)+\Lambda^{T}(s) \cdot J \cdot P(s) \cdot \Lambda(s) & =0, \\
\Lambda^{T}(s)\left[P^{T}(s) \cdot J+J \cdot P(s)\right] \Lambda(s) & =0 .
\end{aligned}
$$

Since $|\Lambda(s)|= \pm 1, \Lambda(s)$ is nonsingular and

$$
J \cdot P(s)=-P^{T}(s) \cdot J=-(J \cdot P(s))^{T}
$$

so that $J \cdot P(s)$ is skew.

Upon differentiating $\Lambda(s) \cdot J \cdot \Lambda^{T}(s)=J$, we obtain similarly

$$
\Lambda^{\prime}(s)=\Lambda(s) \cdot Q(s)
$$

where $Q(s) \cdot J$ is skew.

Clearly $P(s) \cdot J$ is skew if and only if $J \cdot P(s)$ is skew, and similarly $Q(s) \cdot J$ is skew if and only if $J \cdot Q(s)$ is skew.

Since for every $s$ in the interval $s_{0} \leqq s \leqq s_{1},|\Lambda(s)|= \pm 1$, it follows that $\Lambda^{\prime}(s) \cdot \Lambda^{-1}(s)=P(s)$ so that $P(s)$ is unique. Similarly $Q(s)$ $=\Lambda^{-1}(s) \cdot \Lambda^{\prime}(s)$ is unique.

THEOREM 3. Let $P(s)$ be a matrix whose elements are continuous real functions of $s$ in the interval $s_{0} \leqq s \leqq s_{1}$ having the property that $P(s) \cdot J$ is skew. Let $\Lambda(s)$ be a matrix which satisfies a differential equation

$$
\Lambda^{\prime}(s)=P(s) \cdot \Lambda(s),
$$

subject to the initial condition that $\Lambda\left(s_{2}\right) \in \mathbb{R}_{J}$ for some value $s_{2}$ of $s$ in the interval $s_{0} \leqq s \leqq s_{1}$. Then $\Lambda(s) \in R_{J}$ throughout the interval.

Set $\Lambda^{r}(s) \cdot J \cdot \Lambda(s)=R(s)$. Then as before 


$$
\Lambda^{T}(s)\left[P^{T}(s) \cdot J+J \cdot P(s)\right] \Lambda(s)=R^{\prime}(s)
$$

Since $J \cdot P(s)$ is skew, $R^{\prime}(s)=0$ and $R(s)$ is a constant matrix. That is, $R(s)$ has for every value of $s$ in the interval the value that it has for $s=s_{2}$, namely $J$. Thus $\Lambda(s) \in \mathbb{R}_{J}$.

\section{REFERENCES}

1. C. de la Vallee Poussin, Cours d'analyse infinitesimale, Louvin, 1937, p. 259.

2. S. Lefschetz, Lectures on differential equations, Princeton University Press, 1946, chap. III.

3. W. Hurewicz, Ordinary differential equations in the real domain with emphasis on geometric methods, Providence, 1943, p. 39.

4. G. Sansone, Equazioni differenziali nel campo reale, part 1, 2d ed., Bologna, 1948, p. 131.

5. R. A. Frazer, W. J. Duncan, and A. R. Collar, Elementary matrices, Cambridge, 1938, p. 222.

ThE UnIVERSITY OF Wisconsin 